\title{
Clinical Profile of Hepatorenal Syndrome: A Prospective Study
}

\author{
Amit Bery ${ }^{1}$, Rajoo Singh Chhina ${ }^{2}$, Hardeep Sahota ${ }^{1}$, Sandeep Puri ${ }^{1}$, Jaswinder Singh Sandhu ${ }^{3}$, \\ Rajdeep Singh Chhina ${ }^{4}$
}

\author{
Departments of ${ }^{1}$ Medicine, ${ }^{2}$ Gastroenterology, ${ }^{3}$ Nephrology, ${ }^{4}$ Preventive and Social Medicine \\ Dayanand Medical College \& Hospital, Ludhiana, India
}

\begin{abstract}
Background and Objectives : The clinical profile of hepatorenal syndrome (HRS) patients admitted in a tertiary care hospital of Punjab was studied.

Methods : In this prospective study all the patients of chronic liver disease with renal involvement fulfilling the International Ascites Club criteria of HRS were evaluated over a period of one and a half year.

Result : Forty-two patients were diagnosed to have HRS and were included in the study. The incidence of HRS was $0.275 \%$ of hospital medical admissions. Alcoholic cirrhosis was the etiology in $71.5 \%$ of patients. Most of the patients of HRS received a combination of dopamine, albumin and terlipressin. The mortality rate was found to be approximately $60 \%$. Variables amongst survival versus non-survival groups were analyzed. Oliguria and hepatic encephalopathy were more predominant in non-survival group. Serum bilirubin, hypoalbuminemia, hyponatremia, coagulopathy and urine osmolality was higher in non-survival group. Patients with Child-Pugh Score less than 10 had a better survival.

Conclusion : The poor prognostic factors in non-survival group were presence of ascites, severe jaundice, hepatic encephalopathy, alcohol abuse, hypoalbuminemia, progressive renal failure and a Child-Pugh Score > 10. Thus, HRS is not uncommon and needs proper diagnosis and prompt treatment to ensure better outcome.
\end{abstract}

Keywords : Hepatorenal syndrome, outcome, survival

\section{INTRODUCTION}

A wide array of circulatory changes occur in patients with liver cirrhosis and portal hypertension. Hepatorenal syndrome (HRS) is the development of renal failure in patients with advanced liver cirrhosis, who have portal hypertension and ascites, in the absence of kidney disease. The distinctive hallmark feature of HRS is the intense renal vasoconstriction caused by interactions between systemic and portal hemodynamics. This results in activation of vasoconstrictors and suppression of vasodilators in the renal circulation. ${ }^{1}$ Histologically, kidneys are normal and kidney function is reversible with treatment. Although HRS usually occurs in patients with advanced cirrhosis, it has also been described in patients

\section{Corresponding Author :}

Dr. Amit Bery,

Assistant Professor,

Department of Medicine,

Dayanand Medical College \& Hospital,

Ludhiana, Punjab, India

Email: amitbery@yahoo.co.in without ascites in the setting of acute fulminant hepatic failure. $^{2}$

During the late $19^{\text {th }}$ century, Frerichi and Flint made the original description of renal function disturbances in liver disease. In 1950's the clinical description of hepatorenal syndrome by Sherlock, Popper and Vessin emphasized the functional nature of the syndrome, the coexistence of systemic circulatory abnormalities and its dismal prognosis.

In 1996, the International Ascites Club described two different forms of HRS, type 1 and type 2. Although their pathophysiology is similar, but their manifestations and outcomes are different. ${ }^{3}$

Type 1 HRS is characterized by rapid doubling of serum creatinine to a level greater than $2.5 \mathrm{mg} / \mathrm{dl}$ or a halving of the creatinine clearance to less than $20 \mathrm{ml} / \mathrm{min}$ within two weeks and is precipitated most commonly by spontaneous bacterial peritonitis (SBP). It occurs in approximately $25 \%$ of patients with SBP, despite rapid resolution of the infection with antibiotics. Without treatment, median survival of patients with type I 
hepatorenal syndrome is less than 2 weeks and virtually all patients die within 10 weeks after the onset of renal failure. $^{3}$

Type 2 HRS is characterized by a moderate and stable reduction in the glomerular filteration rate (with serum creatinine increasing to $>1.5 \mathrm{mg} / \mathrm{dL}$ or a creatinine clearance less than $40 \mathrm{ml} / \mathrm{min}$ ). It most commonly occurs in patients with relatively preserved hepatic functions and median survival is 3-6 months. Although this is markedly longer than type $1 \mathrm{HRS}$, it is still shorter as compared to patient with cirrhosis and ascites who do not have renal failure. $^{4}$

Watt et $a^{5}$ found that the most common predisposing factor for HRS was bacterial infection (48\%), GI bleed (33\%) and aggressive paracentesis (27\%). Drugs (7\%) and surgery $(7 \%)$ were the precipitating causes. Twenty four percent $(24 \%)$ of the patients with the HRS developed renal failure without an obvious cause.

Moreau et al ${ }^{6}$ observed in 355 patients with cirrhosis and acute renal failure that $58 \%$ patients had pre-renal failure, one third of the patients had type $1 \mathrm{HRS}$, acute tubular necrosis in $41.7 \%$ and $1 \%$ had post renal (obstructive) acute renal failure. Until the recent development of effective therapies, the median survival following the development of type $1 \mathrm{HRS}$ was 1.7 weeks, with $10 \%$ of the patients surviving more than 10 weeks. Survival rates in type two HRS is $50 \%$ at five years and $20 \%$ at one year. The most important aspect in the management of HRS is to prevent its recurrence. The latter is achieved by avoidance, prophylaxis, early recognition and treatment of precipitating factor. The data on profile of HRS from North India is scanty so this prospective study was undertaken to find the clinical profile of HRS.

\section{MATERIAL AND METHODS}

Forty-two (42) patients fulfilling the international ascites club criteria ${ }^{1}$ of HRS were included in the study. The diagnosis of cirrhosis was based on history, examination, liver function tests, ultrasound abdomen and endoscopy. Etiology of cirrhosis was also noted. In all patients a history of jaundice, fever, abdominal pain, abdominal distension, decreased urine output, gastrointestinal bleed and altered sensorium was taken. Ascitic tap and blood cultures were taken to exclude gram negative septicemia and spontaneous bacterial peritonitis. Various variables were studied amongst the survivors and non-survivors to detect possible predictors of survival in HRS. A combination
Table I

Etiology of Chronic Liver Disease in HRS Patients
ETIOLOGY

1. Alcohol

2. Post viral hepatitisa.

HCV Relatedb.

HbsAg Related
NO. OF PATIENTS (\%)

$30(71.5)$

$10(23.8) 02(4.8)$ therapy of dopamine (1-5 $\mu \mathrm{g} / \mathrm{min})$, albumin and terlipressin ( $2 \mathrm{mg} \mathrm{I} / \mathrm{V}$ 6hrly) was used in patients of HRS. Patients were followed up until their discharge or death. Institute's ethical committee approved this study.

\section{STATISTICAL METHODS}

The statistical methods included were the mean, percentage, standard deviation and students " $\mathrm{t}$ " test of significance.

\section{RESULTS}

Forty-two patients with HRS were included in our study. The incidence of HRS was $0.275 \%$ of hospital admissions. In the study, we found $95 \%$ male preponderance. They were all above 30 years of age with mean age of $47.7 \pm 9.8$ years. Alcoholic cirrhosis constituted the commonest etiology of liver disease (71.5\%) (Table I). The viral serology was positive in $28.5 \%$ of the patients, anti-HCV Ab in $23.8 \%$ and HBsAg in $4.8 \%$.

The commonest symptom was jaundice $(92.8 \%)$ followed by decreased urinary output $(85.7 \%)$ and abdominal distension (71.4\%). Altered sensorium was present in $61.9 \%$, fever in $40.9 \%$, pain abdomen $(33 \%)$ and gastrointestinal bleed was seen in $25 \%$ of the patients. All the patients on endoscopy showed varices and had ascites on clinical examination. Icterus and asterixis was present in $92.8 \%$ and $74.4 \%$ cases respectively.

The bilirubin levels were 1.7-56 mg/dl (mean $21.6 \pm 13.6 \mathrm{mg} / \mathrm{dl}$ ). SGOT and SGPT were raised in more than $90 \%$ of the patients (Table II). The mean values of peak blood urea and peak serum creatinine were $125 \pm 75.36 \mathrm{mg} / \mathrm{dl}$ and $4.6 \pm 2.4 \mathrm{mg} / \mathrm{dl}$ respectively. Approximately $66.6 \%$ cases showed hyponatraemia. All the patients had bland urine sediment with urinary spot sodium of $<20 \mathrm{meq} / \mathrm{l}$. Urine osmolality was greater than serum osmolality in all the patients (Table III). The 
Table II

Liver Function Tests

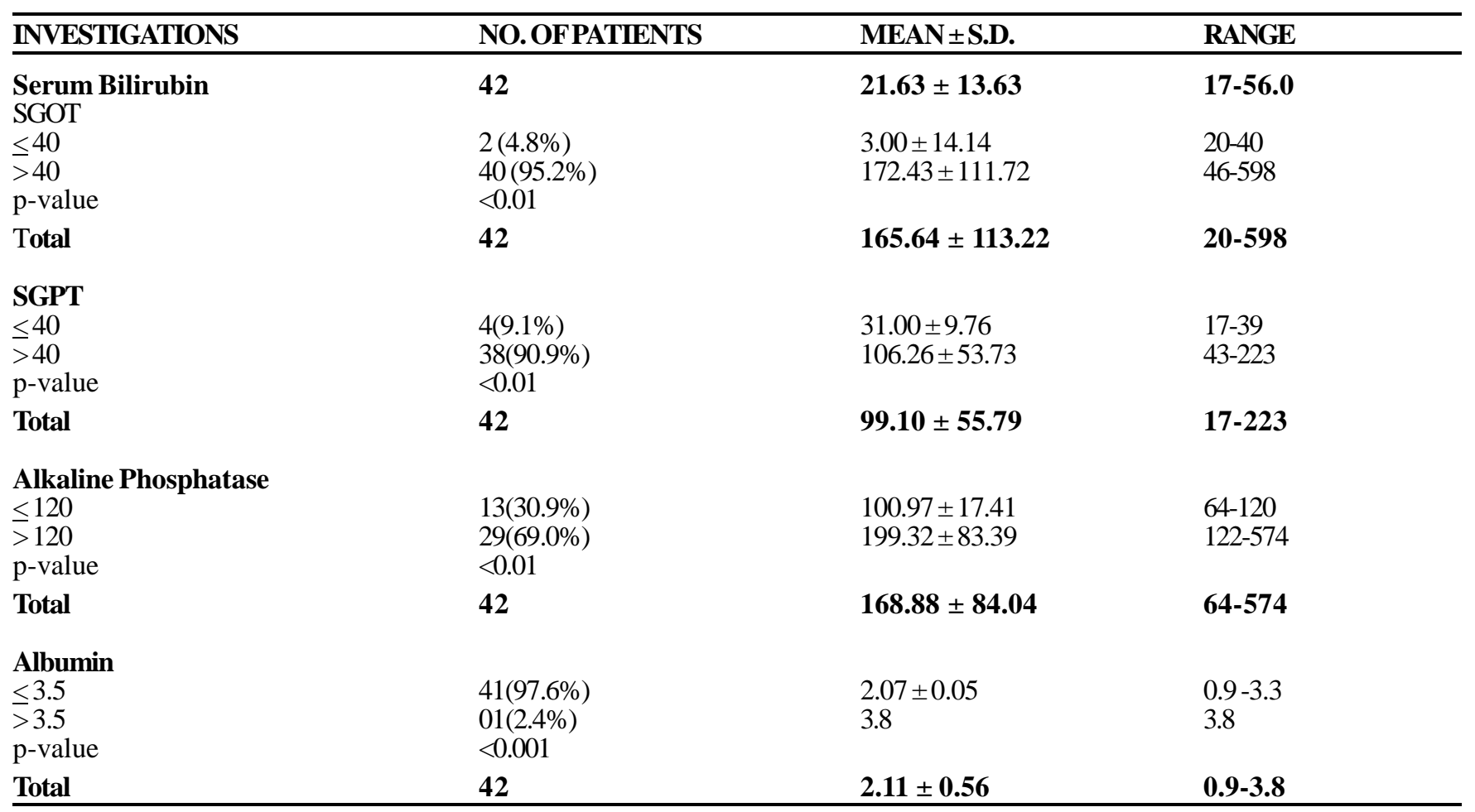

Table III

Renal Function Tests

\begin{tabular}{llll}
\hline INVESTIGATIONS & NO.OFPATIENTS & MEAN \pm S.D. & RANGE \\
\hline $\begin{array}{l}\text { Peak Blood Urea } \\
\text { Peak Serum Creatinine } \\
<1.4\end{array}$ & 42 & $125.50 \pm 75.36$ & $16-332$ \\
$>1.4$ & 0 & 0 & 0 \\
p-value & $42(100)$ & $4.6 \pm 2.4$ & $2.5-10$ \\
\hline $\begin{array}{l}\text { Indices of Renal Failure } \\
\text { Urine Routine }\end{array}$ & $<0.001$ & & \\
$\begin{array}{l}\text { Bland Sediment } \\
\text { Active Sediment }\end{array}$ & 42 & & - \\
\hline $\begin{array}{l}\text { Urine Spot Sodium } \\
<20\end{array}$ & 0 & - & - \\
$>20$ & 42 & - & $1-18$ \\
p-value & 0 & \multicolumn{2}{l}{} \\
Serum Osmolality & 42 & $12.7 \pm 6.3$ & - \\
Urine Osmolality & 42 & - & $235-404$ \\
& & $274.90 \pm 73.55$ & $688-1000$ \\
\hline
\end{tabular}


mortality rate was found to be approximately $60 \%$ (Table IV). The patients who left against medical advice were terminally sick and had very poor prognosis and were included among non-survivors. In our study, about $60 \%$ of patients were treated with combination of albumin infusion, dopamine infusion and terlipressin followed by albumin and dopamine in 33\% patients and dopamine infusion alone in $7 \%$ patients.

Various variables between survival and non-survival groups were compared (Table V). Oliguria was predominant in the non-survival group (96\%). Jaundice was present in all the patients of both the groups. Hepatic encephalopathy was present in $61.9 \%$ of patients, predominantly in the non-survival group. Hypoalbuminaemia and hyponatraemia was more pronounced in non-survival group. Serum bilirubin levels were found to be significantly higher in the non-survival group as compared to survival group. Serum creatinine was found to be insignificantly higher in non-survival group. Ratio of urine osmolality and serum osmolality was higher in non-survival group. Significant number of patients had history of alcohol abuse among the nonsurvivals. Therapy with albumin, dopamine and terlipressin was found to effective in those with Child-Pugh Score $<10$. Jaundice, renal failure, hepatic encephalopathy and ascites were found to be poor prognostic factors with significant $\mathrm{p}$ value. Patients with Child-Pugh Score $<10$ had a better survival advantage.

\section{DISCUSSION}

This prospective study included forty-two patients of HRS. These patients constituted about $0.275 \%$ of medical admission. There was male (95\%) preponderance in our study with mean age of patients of HRS being $47.69 \pm$ 9.75 years. This was similar to studies conducted by Butt et al where mean age of patients was $51.7+11.3$ years and $65 \%$ of patients enrolled were male. ${ }^{7}$ Salerno et al also studied 134 cirrhotics in which the mean age was $61.3+9.6$ years and $73 \%$ of patients were male. ${ }^{8}$

The clinical symptomatology in our patients showed that most of them presented with jaundice (92.9\%), decreased urine output $(85.7 \%)$ and hepatic encephalopathy $(71.4 \%)$. Fever was present in $40 \%$ of patients and gastrointestinal bleed in $32 \%$. These presentations were similar to cases observed by Epstein et $a l^{9}$ where most of the patients had clinical ascites and renal failure at presentation. In our study, we found that all our patients of HRS had ascites, $92.9 \%$ of the patients had icterus, significant percentage had asterixis and history of alcohol abuse. Similar results were seen in studies conducted by Watt et al. ${ }^{5}$

We found raised bilirubin levels (mean value $21.63 \pm$ $13.63 \mathrm{mg} / \mathrm{dL}$ and raised SGOT and SGPT in more than $90 \%$ of the patients. Serum alkaline phosphatase was raised in $69 \%$ of the patients included in the study. Almost all of our patients $(97.6 \%)$ had hypoalbuminaemia. Coagulopathy was present in more than $90 \%$ of the patients. Urine osmolality and serum osmolality ratio was found to be more than one. These findings corroborated with clinical studies conducted by Procel A et al. ${ }^{10}$ Approximately $40 \%$ of HRS cases were discharged from the hospital in satisfactory condition. Remaining either died in the hospital or left against medical advice due to poor prognosis. On comparison of the variables, amongst survivors versus non-survivors, we found that administration of albumin, dopamine and terlipressin to patients who had Child-Pugh Score $<10$ at presentation showed reversal of HRS. Twelve $(64.7 \%)$ patients amongst survivors benefited from the therapy while 52\% of the patients amongst nonsurvivors received this therapy but did not improve and the Child-Pugh score in this group was found to be greater than 10 . The beneficial effect of terlipressin was similar to study conducted by Uriz et $a l^{11}$ and Hadengue et $a l .{ }^{12}$

We compared the various variables in HRS to find the predictive factors and outcome in survival and nonsurvival groups. Oliguria was found in $96 \%$ of the patients of non-survival group, jaundice was present in $100 \%$ of these patients. Higher levels of serum bilirubin (25.09 \pm $13.7 \mathrm{mg} / \mathrm{dL}$ ) were found in non-survivors compared to a mean value of $16.54 \pm 12.19 \mathrm{mg} / \mathrm{dL}$ in survivors. Sixty eight percent of patients who did not survive had hepatic encephalopathy. Decreased levels of albumin were present in non-survivors as compared to survivors. Hyponatraemia was more pronounced amongst the nonsurvival group. Patients in the non-survival group had significant coagulopathy as compared to survivors. The mean urine spot sodium for non-survivors was found to be less as compared to survivors. Urine osmolality and plasma osmolality ratio was found to be greater in the non-survivor group (3.24 \pm 2.16$)$. Significant alcohol abuse was a predominant factor in non-survivors $(84 \%)$.

On statistical analysis, the severity of jaundice, presence of oliguria, hepatic encephalopathy and refractory ascites were poor prognostic factors in patients of HRS. Also Child-Pugh Score > 10 had poor prognostic 
value among the non-survivors. The prognostic value of Child-Pugh Score in HRS was similar to studies conducted by Colle et $a l^{13}$ and Moreau et al. ${ }^{6}$ Once considered fatal with mortality of greater than $90 \%$, in hepatorenal syndrome there is improved prognosis with therapies including terlipressin, dopamine and albumin infusion.

\section{CONCLUSION}

Decompensated alcoholic cirrhosis is the commonest cause of HRS. HRS is not uncommon and with judicious treatment, especially using terlipressin with albumin a significant number of patients can be cured. The poor prognostic factors in HRS are presence of ascites, severe jaundice, hepatic encephalopathy, alcohol abuse, hypoalbuminaemia, progressive renal failure and a ChildPugh Score $>10$.

\section{REFERENCES}

1. Turban S, Thuluvath PJ, Atta MG. Hepatorenal syndrome. World J Gastroenterol 2007;13(30):4046-55.

2. Wilkinson SP, Blendis LM, Williams R. Frequency and type of renal and electrolyte disorders in fulminant hepatic failure. $\mathrm{Br}$ MedJ1974;1:186-9.

3. Santiago JM. The hepatorenal syndrome. Med Clin North Am 2008;92(4):813-37.

4. Arroyo V, Fernandez J, Gines P. Pathogenesis and treatment of hepatorenal syndrome. Semin liver dis 2008;28(1):81-95.

5. Watt K, Uhanova J, Minuk GY. Hepatorenal syndrome:diagnostic accuracy, clinical features, and outcome in a tertiary care centre. Am J Gastroenterol 2002;97(8):2046-50.
6. Moreau R, Durand F, Poynard T, Duhamel C, Cervoni JP, Ichaï $\mathrm{P}$, et al. Terlipressin in patients with cirrhosis and type 1 HRS: A retrospective multicenter study. Gastroenterol 2002;122(4):923-30.

7. Butt AK, Khan AA, Alam A, Shah SW, Shafqat F, Naqvi AB. Predicting hospital mortality in cirrhotic patients: comparison of Child-Pugh and Acute Physiology, Age and Chronic Health Evaluation (APACHE III). Am J Gastroenterol 1998;93(12):2469-75.

8. Solerno F, Barroni G, Moser P, Badalamenti S, Cassarà L, Maggi A, et al. Survival and prognostic factors of cirrhotic patients with ascites; a study of 134 patients. Am J Gastroenterol 1993;88(4):514-9.

9. Epstein M, Berk DP, Hallenberg NK, Adams DF, Chalmers TC, Abrams HL, et al. Renal failure in the patients with cirrhosis; the role of active vasoconstriction. Am J Med 1970;49(2):175-85.

10. Porcel A, Diaz F, Rendon P, Macias M, Martin-Herrera L, GironGonzalez JA. Dilutional hyponatremia in patients with cirrhosis and ascites. Arch Intern Med 2002;162(3):323-8.

11. Uriz J, Gines P, Cardenas A, Sort P, Jimenez W, Salmeron JM, et al. Terlipressin plus albumin infusion: an effective and safe therapy of hepatorenal syndrome. J Hepatol 2000;33(1):43-8.

12. Hadengue A, Gadano A, Moreau R, Giostra E, Durand F, Valla $\mathrm{D}$, et al. Beneficial effects of the 2-day administration of terlipressin in patients with cirrhosis and hepatorenal syndrome. J Hepatol 1998;29(4):565-70.

13. Colle I, Durand F, Pessione F, Rassiat E, Bernuau J, Barriere E, et al. Clinical course, predictive factors and prognosis in patients with cirrhosis and type 1 hepatorenal syndrome treated with terlipressin: a retrospective analysis. J Gastroenterol Hepatol 2002;17(8):882-8. 


\section{PDF created with pdfFactory Pro trial version www.pdffactory.com}

